\title{
Robust Stabilization of the Wave Equation Against Small Delays
}

\author{
Ömer Morgül \\ Department of Electrical and Electronics Engineering \\ Bilkent University \\ 06533, Bilkent, Ankara, TURKEY \\ e-mail : morgul@ee.bilkent.edu.tr
}

\begin{abstract}
In this paper we consider a system which can be modeled by (undamped) wave equation in a bounded domain. We assume that the system is fixed at one end and is controlled by a boundary controller at the other end. We also considered two damped versions of this system, both parametrized by a nonnegative damping constant. We study two poblems for these models, namely the stabilization by means of a boundary controller, and the stability robustness of the closed-loop system against small time delays in the feedback loop. We propose a class of finite dimensional dynamic boundary controllers to solve these problems. One basic feature of these controllers is that the corresponding controller transfer functions are required to be strictly positive real functions. We show that these controllers stabilize both damped and undamped models and solve the stability robustness problem for the damped models. It is also shown that while strict positive realness of the controller transfer functions is important for closed-loop stability, the strict properness is important for the stability robustness against small time delays in the feedback loop.
\end{abstract}

\section{Introduction}

In recent years boundary control of flexible systems has been a very active area of research. Most of the research in this area is concentrated on the problem of control and stabilization of conservative linear flexible systems, (e.g. strings or beams without damping). Such systems have infinitely many eigenvalues on the imaginary axis and can be stabilized by using simple boundary feedback laws. For example, consider the following simple wave equation :

$$
w_{t t}=w_{x x}, \quad 0<x<1, t \geq 0,
$$

$$
w(0, t)=0, w_{x}(1, t)=v(t), y(t)=w_{t}(1, t),
$$

where $v(t)$ is the boundary control input, $y(t)$ is the measured output. The equations (1) and (2) may model a lot of physical systems, such as strings, cables, torsional motion of beams, etc. For simplicity and without loss of generality some constants are chosen to be unity. The equation (2) then is the balance of the internal force $w_{x}(1, t)$ with the applied boundary control input $v(t)$. To control the system given by (1)-(2), one may propose the following simple controller

$$
v(t)=k u(t) \quad, \quad k>0,
$$

where $u$ is the controller input. It is well known that if the following simple feedback law is applied

$$
u(t)=-y(t) \quad,
$$

then the closed loop system given by (1)-(4) becomes exponentially stable, see e.g [2].
Although the controller given by ( 3 ) is quite simple, one of its drawbacks is that the system given by (1)-(4) is not robustly stable with respect to small time delays in the feedback loop. That is if the feedback law (4) is replaced by

$$
u(t)=-y(t-h)
$$

where $h>0$, then the system given by (1)-(3) and (5) becomes unstable for arbitrary small values of $h>0$, see e.g. [4]. This is quite important from a practical point of view since small time delays inevitably occur in any practical applications. Moreover for $k>1$ the situation is even worse in the sense that for any $R>0$, the system (1)-(3) and (5) has an eigenvalue $\lambda$ satisfying $\operatorname{Re}\{\lambda\}>R$ provided that the delay $h>0$ is sufficiently small, see [5].

In this paper we consider the undamped wave equation given by (1) and (2), together with two different damped version of it. To stabilize these systems we propose a dynamic boundary control law. Following [6], we try to answer the following questions :

i : Does the proposed control law stabilize the conservative model and improve the stability of the damped models ?

ii : Does the proposed control law robustly stabilize the damped models against small time delays in the feedback loop?

In the following section we propose a class of dynamic boundary controllers to solve these problems. One basic feature of these controllers is that their transfer functions are required to be strictly positive real functions. This class contain controllers which have strictly proper transfer functions, and this is important from a practical realization of such controllers. Moreover, for such controllers the openloop transfer function of the system may become strictly proper, which is important for the well-posedness of such systems from a control point of view, see [7].

This paper is organized as follows. In section 2 , we give two examples of the damped version of the undamped wave equation given above and propose a class of dynamic controllers to solve the problems stated above, in section 3 we give stability results (i.e. answer to the problem $i$ ), and in section 4 we give robustness results (i.e. answer to the problem ii ). Finally we give some concluding remarks.

\section{Damped Models}

We first consider the following damped wave equation :

$$
\begin{aligned}
& w_{t t}=w_{x x}-2 a w_{t}-a^{2} w, 0<x<1, t \geq 0, \\
& w(0, t)=0, w_{x}(1, t)=v(t), y(t)=w_{t}(1, t),
\end{aligned}
$$

where $a \geq 0$ is a damping constant, $v(t)$ is the boundary control input and $y(t)$ is the measured output. 
The system given by (6)-(7) is first introduced in [4], and later investigated in [1], [10] and [6]. For $a=0$, the system given by (6), (7) reduces to the system given by (1), (2). It is known that the controller given by (3) and the feedback law given by (4) exponentially stabilizes the system (6), (7) see [4]. The stability of (6), (7) with the feedback law (5) depends on $k$ and $a$. It is known that if

$$
k>\frac{1-e^{-2 a}}{1+e^{-2 a}}
$$

then the closed loop system (5)-(7) is unstable for arbitrary small time delays $h>0$. On the other hand if

$$
k<\frac{1-e^{-2 a}}{1+e^{-2 a}},
$$

then there exists an $h_{0}>0$ such that for any $h, 0 \leq h \leq h_{0}$ the system (5)-(7) is $L_{2}$-stable. This result is obtained in [4] by calculating the eigenvalues directly and in [1] and [10] by using frequency domain techniques.

The second example of the damped wave equation that we consider is the following :

$$
w_{t t}=w_{x x}+a w_{x x t}, 0<x<1, t \geq 0,
$$

$w(0, t)=0, w_{x}(1, t)+\alpha a w_{x t}(1, t)=v(t), y(t)=w_{t}(1, t)$

where $a \geq 0 \mathrm{~s}$ a damping constant, $\alpha$ is either 0 or 1 . This type of damping is not unnatural, (see [9]), and is similar to Kelvin-Voight damping for the Euler-Bernoulli beam. The system (10), (11), with $\alpha=0$, is first introduced and inves. tigated in [6]. It can be shown that with the controller given by (3) and the feedback law given by (4) the closed loop is exponentially stable, (see Theorem 2 in section 3 ). However, it was shown in [6] by direct eigenvalue calculations that the closed loop system becomes unstable for arbitrary time delays $h>0$ when (4) is replaced by (5). Moreover, it was shown in [6] that in this case for any $R>0$ and $h_{0}>0$, there exists an $h>0$ such that $0<h<h_{0}$ and an eigenvalue $\lambda$ of $(10),(11)$ and (5) satisfying $R e\{\lambda\}>R$. In section 4 we will show that this instability could be predicted by considering the open loop transfer function and could be eliminated by choosing $\alpha=1$, (see Corollary 2 in section 4 ). We note that the case $\alpha=1$ gives the natural boundary condition for (10), and this can be justified by considering the rate of change of the energy of the system. For a comparison with similar boundary conditions for the Euler-Bernoulli beam with Kelvin-Voight damping. In section 4 we will show that even in the case $\alpha=0$, by choosing appropriate dynamic boundary controllers, the instability with respect to time delays can be eliminated, (See corollary 2).

We propose the following dynamic boundary controllers to solve the stability problems stated above :

$$
\begin{gathered}
\dot{z}=A z+b u, \\
v=c^{T} z+d u,
\end{gathered}
$$

where $z \in \mathbf{R}^{n}$, for some natural number $n$, is the controller state, $u$ is the controller input, $A \in \mathbf{R}^{n \times n}$ is a constant matrix, $b, c \in \mathbf{R}^{n}$ are constant column vectors, $d$ is a constant real number and the superscript $T$ denotes transpose.

We first make the following assumptions concerning the controller given by (12), (13) thoroughout this work :

Assumption 1 : All ejgenvalues of $A \in \mathbf{R}^{n \times n}$ have negative real parts.
Assumption $2:(A, b)$ is controllable and $(c, A)$ is observable.

Assumption $3: d \geq 0$, and there exists a constant $\gamma$ $d \geq \gamma \geq 0$, such that the following holds

$d+\operatorname{Re} e\left\{c^{T}(j \omega I-A)^{-1} b\right\}>\gamma, \quad, \quad \omega \in \mathbf{R}$,

moreover, in case $d>0$, we require $\gamma>0$ as well. $\square$

We note that this type of controllers have been proposed for the stabilization of flexible structures. For the application to wave equation, see [11], [13], to the Euler-Bernoulli beam, see [12], and to the rotating flexible structures, see [14].

If we take the Laplace transform in (12) and (13) and use zero initial conditions, we obtain :

$$
\hat{v}(s)=g(s) \hat{u}(s)=\left[d+c^{T}(s I-A)^{-1} b\right] \hat{u}(s),
$$

where a hat denotes the Laplace transform of the corresponding variable. This, together with (14) implies that the transfer function $g(s)$ in (15) is a strictly positive real function, see [16].

Assume that the controller is given by a transfer function $g(s)$. One can always find a minimal realization $(A, b, c, d)$ for $g(s)$, which satisfy (15). Because of minimality, the eigenvalues of $A$ are the same as the poles of $g(s)$. Hence, in case the actuator is given by a proper transfer function $g(s)$, as in (15), as opposed to the state-space representation given by (12), (13), an equivalent characterization of the assumptions $1-3$ are :

Assumption i : All poles of $g(s)$ have negative real parts.

Assumption ii : There exists a $\gamma \geq 0$ such that the following holds :

$$
\operatorname{Re}\{g(j \omega)\}>\gamma \quad, \quad \omega \in \mathbf{R} \square .
$$

\section{Stability Results}

Let the assumptions 1-3, or equivalently the assumptions $\mathrm{i}-\mathrm{i}$ stated above hold. Then, it follows from the Meyer-KalmanYakubovich Lemma that given any symmetric positive definite matrix $Q \in \mathbf{R}^{n \times n}$, there exists a symmetric positive definite matrix $P \in \mathbf{R}^{n \times n}$, a vector $q \in \mathbf{R}^{n}$ and a constant $\epsilon>0$ satisfying :

$$
\begin{gathered}
A^{T} P+P A=-q q^{T}-\epsilon Q, \\
P b-c=\sqrt{2(d-\gamma)} q,
\end{gathered}
$$

see $[16$, p. 133]. In case $d=0$ in (13), we can take $q=0$ and $\epsilon=1$, see [16, p. 132].

To analyze the systems considered in this paper, we first define the function space $\mathcal{H}$ as follows

$$
\mathcal{H}:=\left\{(p q z)^{T} \mid p \in \mathbf{H}_{\mathbf{0}}^{1}, q \in \mathbf{L}^{2}, z \in \mathbf{R}^{n}\right\},
$$

where the spaces $\mathrm{L}^{2}$ and $\mathrm{H}_{0}^{k}$ are defined as follows :

$$
\begin{gathered}
\mathbf{L}^{2}=\left\{f:[0, L] \rightarrow \mathbf{R} \mid \int_{0}^{L} f^{2} d x<\infty\right\} \\
\mathbf{H}_{0}^{k}=\left\{f \in \mathbf{L}^{2} \mid f, f^{\prime}, f^{\prime \prime}, \ldots, f^{(k)} \in \mathbf{L}^{2}, \quad f(0)=0\right\}
\end{gathered}
$$


The equations (6), (7), (12) and (13) together with the feedback control law (4) can be written in the following abstract form :

$$
\dot{m}=\mathcal{A}_{1} m \quad, \quad m(0) \in \mathcal{H},
$$

where $m=\left(w w_{t} z\right)^{T} \in \mathcal{H}$, the operator $\mathcal{A}_{1}: \mathcal{H} \rightarrow \mathcal{H}$ is a linear unbounded operator defined as

$$
\mathcal{A}_{\mathbf{1}}\left(\begin{array}{c}
p \\
q \\
z
\end{array}\right)=\left(\begin{array}{c}
q \\
p_{x x}-2 a q-a^{2} p \\
A z+b q(1)
\end{array}\right) .
$$

The domain $D\left(\mathcal{A}_{1}\right)$ of the operator $\mathcal{A}_{1}$ is defined as :

$$
\begin{aligned}
D\left(\mathcal{A}_{1}\right):= & \left\{(p q z)^{T} \in \mathcal{H} \mid p \in \mathbf{H}_{0}^{2}, q \in \mathbf{H}_{0}^{1}, z \in \mathbf{R}^{n} ;\right. \\
& \left.p_{x}(1)+c^{T} z+d q(1)=0\right\}
\end{aligned}
$$

Let the assumptions 1-3 hold, let $Q \in \mathbf{R}^{n \times n}$ be an arbitrary symmetric positive definite matrix and let $P \in \mathbf{R}^{n \times n}$, $q \in \mathbf{R}^{n}$ be the solutions of (17) and (18) where $P$ is also a symmetric and positive definite matrix. In $\mathcal{H}$, we define the following "energy" inner-product:

$$
<m, \tilde{m}>_{E}=\frac{1}{2} \int_{0}^{1} q \tilde{q} d x+\frac{1}{2} \int_{0}^{1} p_{x} \tilde{p}_{x} d x+\frac{1}{2} \tilde{z}^{T} P z,
$$

where $m=(p q z)^{T}, \tilde{m}=(\tilde{p} \tilde{q} \tilde{z})^{T} \in \mathcal{H}$. We note that $\mathcal{H}$, together with the energy inner-product given by (25) becomes a Hilbert space. The "energy" norm induced by $(25)$ is :

$$
E(t):=\|m(t)\|_{E}^{2}=\frac{1}{2} \int_{0}^{1} w_{t}^{2} d x+\frac{1}{2} \int_{0}^{1} w_{x}^{2} d x+\frac{1}{2} z^{T} P z
$$

Theorem 1 : Consider the system given by (22), where the operator $\mathcal{A}_{1}$ is given by (23). Assume that the assumptions $1-3$ are satisfied.

i : The operator $\mathcal{A}_{1}$ generates a $C_{0}$-semigroup $T(t)$ of contractions in $\mathcal{H}$, (for the terminology of the semigroup theory, the reader is referred to e.g. [15]).

ii : For $a=0, d=0$, the semigroup $T(t)$ is asymptotically stable, i.e. the solutions of (22) asymptotically converge to 0 .

iii : For $a+d>0$, the semigroup $T(t)$ is exponentially stable.

Proof :

i : We first define the following new "energy" of the system :

$$
E_{1}(t)=E(t)+\frac{a^{2}}{2} \int_{0}^{1} w^{2} d x \quad,
$$

where $E(t)$ is given by (26). Note that due to the boundary condition (7) at the fixed end, the integral term in (27) can be embedded in $E(t)$. By differentiating (27) with respect to time, we obtain :

$$
\begin{aligned}
\dot{E}_{1}(t)= & y v-2 a \int_{0}^{1} w_{t}^{2} d x+\frac{1}{2} z^{T}\left(A^{T} P+P A\right) z+z^{T} P b u \\
= & -2 a \int_{0}^{1} w_{t}^{2} d x-\gamma w_{t}^{2}(1, t) \\
& -\frac{1}{2}\left[\sqrt{2(d-\gamma)} w_{t}(1, t)+z^{T} q\right]^{2}-\frac{\epsilon}{2} z^{T} Q z
\end{aligned}
$$

where in deriving the first equation we used integration by parts, (6), (7), (12) and (13), to obtain the second equation we used (4), (17) and (18). It follows from (28) that the operator $\mathcal{A}_{1}$ is dissipative. It can be shown that the operator $\lambda I-\mathcal{A}_{1}: \mathcal{H} \rightarrow \mathcal{H}$ is onto for $\lambda>0$, (see [11], [13] for similar calculations). Hence from Lumer-Phillips theorem we conclude that $\mathcal{A}_{1}$ generates a $C_{0}$-semigroup of contractions on $\mathcal{H}$, see [15].

ii : See [11], [13]

iii : For $a=0$ and $d>0$, see [11]. Hence we consider the case $a>0, d \geq 0$. It is known that the operator $\mathcal{A}_{1}$ has compact resolvent when $a=0$, see [11]. Since the terms containing $a$ can be considered as a bounded perturbation to this operator, it can easily be shown that the operator $\mathcal{A}_{1}$ has compact resolvent for $a>0$ as well. This implies that the operator $\mathcal{A}_{1}$ has point spectrum. By using (28) it can be shown that $\mathcal{A}_{1}$ cannot have an eigenvalue on the imaginary axis. Since $\mathcal{A}_{1}$ has point spectrum, it follows that the imaginary axis belongs to the resolvent of $\mathcal{A}_{1}$.

To obtain an estimate of the resolvent on the imaginary axis, let $y=(f h r)^{T} \in \mathcal{H}$ be given. We have to find $m=(p q z)^{T} \in D\left(\mathcal{A}_{1}\right)$ such that :

$$
\left(j \omega I-\mathcal{A}_{1}\right) m=y
$$

By using (23) in (29), after some straightforward calculations we conclude that $\left\|\left(j \omega I-\mathcal{A}_{1}\right)^{-1}\right\|<\infty$ for $\omega$ sufficiently large, (see [11] for similar estimates). Since the imaginary axis belongs to the resolvent set $\rho\left(\mathcal{A}_{1}\right)$ of the operator $\mathcal{A}_{1}$, and since for each $\lambda \in \rho\left(\mathcal{A}_{1}\right)$, the operator $(\lambda I-L)^{-1}$ is compact, it follows that for any $\Omega<\infty$, the following estimate holds :

$$
\sup _{\omega \leq \Omega}\left\|\left(j \omega I-\mathcal{A}_{1}\right)^{-1}\right\|<\infty .
$$

By combining these results we conclude that the estimate (30) holds for all $\omega$. Hence, it follows from a result of [8] that the $C_{0}$-semigroup $T(t)$ generated by the operator $\mathcal{A}_{1}$ is exponentially stable. $\square$

Now we consider the system given by (10), (11), with the controller (12), (13) and the feedback law (4). This system can be written in the following abstract form :

$$
\dot{m}=\mathcal{A}_{2} m \quad, \quad m(0) \in \mathcal{H},
$$

where $m=\left(w w_{t} z\right)^{T} \in \mathcal{H}$, the operator $\mathcal{A}_{2}: \mathcal{H} \rightarrow \mathcal{H}$ is a linear unbounded operator defined as

$$
\mathcal{A}_{2}\left(\begin{array}{c}
p \\
q \\
z
\end{array}\right)=\left(\begin{array}{c}
q \\
p_{x x}+a q_{x x} \\
A z+b q(1)
\end{array}\right),
$$

where $(p q z)^{T} \in \mathcal{H}$. The domain $D\left(\mathcal{A}_{2}\right)$ of the operator $\mathcal{A}_{2}$ is defined as :

$$
\begin{aligned}
D\left(\mathcal{A}_{2}\right):= & \left\{( p q z ) ^ { T } \in \mathcal { H } \left(p \in \mathbf{H}_{0}^{2}, q \in \mathbf{H}_{0}^{2}, z \in \mathbf{R}^{n}\right.\right. \\
& \left.p_{x}(1)+c^{T} z+d q(1)=0\right\}
\end{aligned}
$$

Theorem 2 : Consider the system given by (31). Assume that the assumptions $1-3$ are satisfied. Then we have the following.

i : The operator $\mathcal{A}_{2}$ generates a $C_{0}$-semigroup $T(t)$ of contractions for each one of the following cases :

i.1 : for $\alpha=1$,

i.2 : for $\alpha=0$ and $d=0$,

i.3 : for $\alpha=0$ and $d>0$, provided that $c^{T} b$ is sufficiently large or $a$ is sufficiently small.

ii : For $a=0, d=0$, the semigroup $T(t)$ is asymptotically stable, i.e. the solutions of (31) asymptotically converge to 0 . 
: For $a+d>0$, the semigroup $T(t)$ is exponentially stable.

Proof :

i : For the case i.1, consider the "energy" $E(t)$ given by (26). By differentiating (26) with respect to time, and by using (4), (10)-(13), it can be shown that $\dot{E} \leq 0$, hence the operator $\mathcal{A}_{2}$ is dissipative for the case i.1.

For the case i.2, we again consider the "energy" $E(t)$ given by (26). Note that in this case since $d=0$, without loss of generality we can take $q=0$ and $\epsilon=1$ in (17) and (18), see [16, p. 132]. By differentiating (26) with respect to timle, and by using (4), (10)-(13), and by using some

straightforward inequalities it can be shown that $\dot{E} \leq 0$, hence the operator $\mathcal{A}_{2}$ is dissipative for the case i.2.

Finally, for the case i.3, we choose the following "energy" function $E_{2}(t)$

$$
E_{2}(t)=E(t)+\frac{1}{2} a d w_{t}^{2}(1, t),
$$

where $E(t)$ is given by (26). By differentiating (34) with respect to time and by using (4), (10)-(13), and following the analysis for the case i.2 it can be shown that $\dot{E}$ can be made negative if $a$ is sufficiently small or $c^{T} b$ is sufficiently large. Provided that, we conclude that operator $\mathcal{A}_{2}$ is dissipative for the case i.3.

It can easily be shown that in all cases the operator $\lambda I-$ $\mathcal{A}_{2}: \mathcal{H} \rightarrow \mathcal{H}$ is onto for $\lambda>0$, (see [11] for similar results). It then follows from the Lumer-Phillips Theorem that the operator $\mathcal{A}_{2}$ generates a $C_{0}$ semigroup of contractions in $\mathcal{H}$.

ii : See [11]

iii : The case $a=0, d>0$ was proved in [11]. Hence, we consider the case $a>0$ and $d>0$. It is known that for the uncontrolled case (i.e (10) and (11) with $v \equiv 0$ ), the resulting system generates an exponentially decaying analytic semigroup, see [9]. Since the controller given by (12) and (13) is essentially finite dimensional, it can be shown that the operator $\mathcal{A}_{2}$ generates an analytic semigroup when $b \equiv 0$. The term multiplying $b$ can be considered as a perturbation and it can easily be shown that $\mathcal{A}_{2}$ generates an analytic semigroup when $\|b\|$ is sufficiently small, (see [ 15 , p. 80-81]). Note that for any $k>0$, we can rescale $b$ and $c$ as $\bar{b}=k b, \tilde{c}=\frac{1}{k} c$. without changing the transfer function $g(s)$ given by (15). Hence, without loss of generality we can assume that $\|b\|$ could be selected as small as desired, hence the operator $\mathcal{A}_{2}$ generates an analytic semigroup. Since the semigroup $T(t)$ genterated by $\mathcal{A}_{2}$ is a contraction semigroup, it follows that the imaginary axis belongs to its resolvent set and that the estimate (30) holds for the operator $\mathcal{A}_{2}$, for all $\omega$, see $[15$, p. $61-62]$. Therefore, from $[8]$ it follows that the semigroup $T(t)$ generated by $\mathcal{A}_{2}$ is exponentially decaying.

\section{Robust Stability with Respect to Small Time Delays}

In this section we analyze the stability of the systems (6), (7) and (10), (11) together with the controller (12), (13) and the delayed feedback law (5). To analyze input-output stability of this system, we use the frequency domain approach. The terminology used here is borrowed from [10]. Let $H(s)$ denote the transfer function of an SISO plant between its in- put $u$ and its output $y . H(s)$ is said to be well-posed if it is bounded on some right half plane and is said to be regular if it has a limit at $+\infty$ along the real axis. If we apply the unity feedback and set $u=r-y$, where $r$ is the new input, then the closed-loop transfer function between $r$ and $y$ becomes $G^{0}(s)=H(s)(1+H(s))^{-1}$. When there is a small time delay by $\epsilon$ in the feedback loop, the new transfer function $G^{\epsilon}(s)$ from $r$ to $y$ becomes $G^{\epsilon}(s)=H(s)\left(1+e^{-\epsilon s} H(s)\right)^{-1}$. We say that $G^{0}$ is robustly stable with respect to delays if there is an $\epsilon_{0}>0$ such that for any $\epsilon \in\left[0, \epsilon_{0}\right], G^{\epsilon}$ is $L_{2}$-stable. If this property does not hold, then arbitrary small time delays destabilize $G^{0}$.

Let the transfer function $H(s)$ be meromorphic (i.e. analytic except at its poles) on the half plane $C_{0}=\{s \in$ $\mathbf{C} \mid \operatorname{Re}\{s\}>0\}$. Let $\mathcal{B}$ denote the (discrete) set of poles of $H$ in $C_{0}$, and let $\gamma^{*}$ be defined as :

$$
\gamma^{*}=\lim _{|s| \rightarrow \infty} \sup _{s \in C_{0}-\mathcal{B}}|H(s)|
$$

Theorem 4 : Let $H(s)$ be a regular transfer function and assume that $G^{0}=H(1+H)^{-1}$ is $L_{2}$-stable. Let $\gamma^{*}$ be defined as in (35).

i : If $\gamma^{*}<1$, then $G^{0}$ is robustly stable with respect to delays.

ii : If $\gamma^{*}>1$, then $G^{0}$ is not robustly stable with respect to delays.

Proof : See [10]. For a different version of this result, see $[3] . \square$

Now consider the system given by (6), (7), (12), (13). An easy calculation shows that the (open loop) transfer function $H(s)$ from $u$ to $y$ is :

$$
H(s)=\frac{s g(s)}{(s+a)} \frac{1-e^{-2(s+a)}}{1+e^{-2(s+a)}},
$$

where $g(s)$ is given by (15), (see also [1] and [10] for the case $g(s)=k$, where $k>0$ is a constant). Since the system is exponentially stable for the case $a+d>0$, (see Theorem 1), it follows that $G^{\circ}$ is $L_{2}$-stable, hence Theorem 4 is applicable. Note that when $d=0$, both $g(s)$ and $H(s)$ are strictly proper. As is shown below, this is important for the stability robustness with respect to small delays.

Corollary 1 : Consider the system given by (6), (7), $(12),(13)$. Let the assumptions of Theorem 1 be satisfied Assume that $a>0$.

i : If $d<\frac{e^{2 a}-1}{e^{2 a}+1}$, then $G^{0}$ is robustly stable with respect to time delays

ii : If $d>\frac{e^{2 a}-1}{e^{2 a}+1}$, then $G^{0}$ is not robustly stable with respect to time delays.

Remark 1 : This result has been known for the nondynamic controller case (i.e. when $g(s)=k$, where $k>0$ is a constant), see [4], [1], [10], [6]. Hence Corollary 1 can be considered as a generalization of the similar results presented in the references mentioned above. However, note that Corollary 1 is still valid when $d=0$, in which case the case $\mathbf{i}$ is trivially satisfied, hence the corresponding $G^{\circ}$ is always robustly stable with respect to small time delays for all $a>0$. Moreover by 'Theorem 1 , for the case $d=0$, the closed-loop system is exponentially stable for $a>0$ and is asymptoticaliy stable for $a=0$. Hence, the controller given by (12) and (13) solves the problems stated in the introduction. Moreover, for the case $d=0$, both the corresponding controller transfer function $g(s)$ and the open-loop transfer 
function $H(s)$ are strictly proper, see (15) and (36). These points are important for actual implementation of $g(s)$ and for the well-posedness of the model, see [7]. $\square$

Proof: From the formulation it is obvious that Theorem 4 is applicable, hence we need to compute $\gamma^{*}$ given by (35). Note that $|g(s)|$ is bounded on $C_{0}$ and $g(s)=d+o(1 / s)$ for large $s$. By using this, and the results of [10], it can be shown that

$$
\gamma^{*}=d \frac{e^{2 a}-1}{e^{2 a}+1}
$$

To see this, following [10], first note that for $s \in C_{0}$, we have $\left|1-e^{-2(s+a)}\right| \leq 1+e^{-2 a}$, and

$\left|1+e^{-2(s+a)}\right| \geq 1-e^{-2 a}$, and $|s /(s+a)| \leq 1$. This shows that $\gamma^{*} \leq d \frac{e^{2 a}-1}{e^{2 a}+1}$. To prove the reverse inequality, we choose $s_{n}=1 / n+j(2 n+1) \pi / 2$ for $n \in \mathrm{N}$. It can easily be shown that $\lim _{n \rightarrow \infty} H\left(s_{n}\right)=d \frac{e^{2 a}-1}{e^{2 a}+1}$ which proves that (37) is satisfied. The claims of Corollary 1 now follows from Theorem 4. $\square$

Next, we consider the system given by (10)-(13), An easy calculation shows that the open-loop transfer function from $u$ to $y$ is :

$$
H(s)=\frac{s g(s)}{\beta(1+\alpha a s)} \frac{1-e^{-2 \beta}}{1+e^{-2 \beta}},
$$

where $\beta$ is given by :

$$
\beta^{2}=\frac{s^{2}}{(1+a s)},
$$

and $g(s)$ is given by (15). Since the system is exponentially stable for the case $a+d>0$, (see Theorem 2), it follows that $G^{0}=H(1+H)^{-1}$ is $L_{2}$ - stable, hence Theorem 4 is applicable. We have the following corollary.

Corollary 2 : Consider the system given by (10)-(13). Let the conditions in Theorem 3 are satisfied. Assume that $a>0$.

i : If $\alpha=1$, then $\gamma^{*}=0$, hence $G^{0}$ is robustly stable with respect to small time delays.

ii : If $\alpha=0$ and $d=0$, then $\gamma^{*}=0$, hence $G^{0}$ is robustly stable with respect to small time delays.

iii : If $\alpha=0$ and $d>0$, then $\gamma^{*}=+\infty$, hence $G^{0}$ is not robustly stable with respect to small time delays.

Remark 2 : Example 2 was first introduced in [6]. The controller proposed in [6] was non-dynamic, i.e. (12)-(13) are not present and the controller was given by (3). It can be shown that the conclusions of Theorem 2 and Corollary 2 are valid in this case as well. To see this, note that we should take $d=\gamma=k, A=Q=0, q=b=c=0$, hence it follows from Theorem 2 that the corresponding operator is still dissipative, and the conclusions of $\mathbf{i . 3}$ and iii of Theorem 2 hold in this case (with no assumptions on $c^{T} b$ or a). The corresponding open-loop transfer function is still given by (38) with $g(s)=k$. Hence case iii of Corollary 2 is still valid, i.e. $\gamma^{*}=+\infty$, hence the system is not robustly stable with respect to small time delays. Hence, Theorem 2 and Corollary 2 can be considered as a generalization of similar results presented in [6]. Moreover, as stated in the case ii of Corollary 2 , the use of strictly proper controllers (i.e. $d=0$ ) eliminates the instability due to small time delays. Moreover, by Theorem 3, for the case $d=0$, the closed-loop system is exponentially stable for $a>0$ and is asymptotically stable for $a=0$ both for $\alpha=0$ or 1 . Hence, the controller given by (12) and (13) solves the problems stated in the introduction. Moreover, for the case $d=0$, both the corresponding controller transfer function $g(s)$ and the open-loop transfer function $H(s)$ (for $\alpha=0$ ) are strictly proper, see (15) and (38). As stated in Remark 1, these points are important for actual implementation of $g(s)$ and for the well-posedness of the model, see [7]. This example, as well as example 1 , shows the importance of strictly proper controllers to prove certain stability robustness results. $\square$

Proof: From the formulation it is obvious that Theorem 4 is applicable, hence we need to compute $\gamma^{*}$ given by (35). For $s \in C_{0}$, it follows from (39) that $\beta \in C_{0}$ as well, hence we have $\left|1-e^{-2 \beta}\right| \leq 2$ for $s \in C_{0}$. Next we show that inf $_{s \in C_{0}}\left|1+e^{-2 \beta}\right|>0$. To show this, first we define the set $C_{M}=\left\{s \in C_{0}|| s \mid \geq M\right\}$ for $M>0$. From (39) it follows that for $|s|$ sufficiently large we have $\beta \sim \sqrt{s / a}$, hence one can easily show that $\left|1+e^{-2 \beta}\right| \geq 1-e^{\sqrt{2 M / a}}$ for $s \in C_{M}$, provided that $M$ is sufficiently large. Also an easy calculation shows that all zeroes of $1+e^{-2 \beta}=0$ and (39) are in the left half of the complex plane, and are all bounded away from the imaginary axis. Hence it follows easily that inf $\operatorname{s\in C}_{0}-C_{M}\left|1+e^{-2 \beta}\right|>0$, for otherwise there must be a zero in $C_{0}-C_{M}$, which is a contradiction. From these arguments it follows that $\frac{1-e^{-2 \beta}}{1+e^{-2 \beta}}$ is bounded on $C_{0}$. Since $g(s)$ is bounded on $C_{0}$ and $g(s) \sim d+o(1 / s)$ for $|s|$ sufficiently large, it follows from (35) and (38) that for $\alpha=1$, we have $\gamma^{*}=0$. For the case $\alpha=0$ and $d=0$, note that $g(s) \sim o(1 / s)$ for large $s$, hence we have $\gamma^{*}=0$. For the case $\alpha=0$ and $d>0$, it follows that $\gamma^{*} \sim \sqrt{s}$ for large $s$, hence we have $\gamma^{*}=+\infty$. Now, Corollary 2 follows from Theorem 4. $\square$

\section{Conclusion}

In this paper we considered a system which can be modeled by an undamped (conservative) wave equation in a bounded domain. We also considered two different damped versions of the same system, both parametrized by a damping constant $a \geq 0$. We assumed that the system is fixed at one end and is controlled at the other end. The justification of using both undamped and damped models is that the solutions predicted by the undamped models are often good approximations to the underlying physical systems over a finite time interval. However, in most of the physical systems some sort of internal damping is present and the effect of damping becomes dominant for large time. Hence to make meaningful asymptotical analysis, one has to take the effect of damping into consideration. Therefore it can be argued that any control theory based on undamped models should justify its conclusions by using appropriately damped version(s) of these models, see [6]. Also it can be shown that many undamped models are ill-posed from a control theory point of view and possess potential limitations for use in the feedback design, see [7].

For the models considered in this paper, we studied two problems : stabilization of the system by means of a boundary controller, and stability robustness with respect to small time delays in the feedback loop. To solve these problems, we proposed a class of dynamic boundary controllers. Under some assumptions, one of which is the strict positive realness of the controller transfer functions, we proved the following 
i : The proposed controllers stabilize both damped and undamped models. Moreover, the closed loop system is asymptotically stable for the case $a=0, d=0$, and is exponentially stable for $a+d>0$, where $a \geq 0$ is the damping coefficient and $d \geq 0$ is the controller feedthrough term, see (13). Note that $a=0$ and $a>0$ corresponds to undamped and damped model, respectively, whereas for $d=0$ and $d>0$ the controller transfer function is strictly proper, and proper but not strictly proper, respectively.

ii : The closed-loop system is robustly stable with respect to small time delays in the feedback loop for the case $a>0$ and $d=0$. Moreover, in this case both the controller transfer function and the open-loop transfer function become strictly proper. That the controller transfer function is strictly proper is important from a practical point of view, since most physical controllers are essentially band-limited and hence exhibit strictly proper behaviour, and that the open-loop transfer function is strictly proper is important for the model well-posedness, since otherwise such models possess potential limitations for the feedback design, see [7].

Finally, the ideas presented here can be extended to other flexible structures, such as flexible beams under various modeling assumptions. This will be the subject of a forthcoming paper.

\section{References}

[1] Bontsema, J., and S. A. deVries, "Robustness of Flexible Structures Against Small Time Delays," Proc. of 27 th $C D C$, Austin, Texas, 1988, pp. 1647-1648.

[2] Chen, G., "Energy Decay Estimates and Exact Boundary Value Controllability for the Wave Equation in a Bounded Domain," J. Math. Pures. Appl., vol.58, 1979, pp.249-273.

[3] Barman, J. F., F. M. Callier, and C. A. Desoer, " $L_{2}$ stability and $L_{2}$-instability of Linear Time Invariant Distributed Feedback Systems Perturbed by a Small Delay in the Loop," IEEE Trans. on $A C$, vol. 18, 1973, pp. 479-484.

[4] Datko, R., J. Lagnese, and M. P. Polis, "An Example on the Effect of Time Delays in Boundary Feedback Stabilization of Wave Equations," SIAM J. on Contr. and Optim.,, vol. 24, 1986, pp. 152-156.

[5] Datko, R. "Two Examples of Ill-Posedness with Respect to Small Time Delays in Stabilized Elastic Systems," IEEE Trans. on $A C$, vol. 38, no. 1, 1993, pp. 163-166.

[6] Datko, R. "Two Questions Concerning the Boundary Control of Certain Elastic Systems," J. of Diff. Eqns., vol. 92, 1991, pp. 27-44.
[7] Helmicki, A. J., C. A. Jacobson, and C. N. Nett, "Ill-Posed Distributed Parameter Systems: A Control Viewpoint," IEEE Trans. Auto. Contr., vol. 36, No. 9, 1991, pp. 1053-1057.

[8] Huang, F. L. , "Characteristic Conditions for Exponential Stability of Linear Dynamical Systems in Hilbert Spaces," Annales Diff. Equations. vol 1, No. 1, 1985, pp. 43-53.

[9] Massatt, P. "Limiting Behaviour for Strongly Damped Non-Linear Wave Equations," J. Diff. Eqns., vol. 48, 1983, pp. 334-349.

[10] Logemann, H., R. Rebarber, and G. Weiss, "Conditions for Robustness and Nonrobustness of the Stability of Feedback Systems with Respect to Small Delays in the Feedback Loop," Report no. 285, Institut für Dynamische Systerne, Universitat Bremen, June 1993.

[11] Morgül, Ö., "A Dynamic Control Law for the Wave Equation," Automatica, Nov. 1994.

[12] Morgül, Ö., "Dynamic Boundary Control of a EulerBernoulli Beam," IEEE Trans. on Auto. Contr., vol 37 , No. 5, 1992, pp. 639-642.

[13] Morgül, Ö., "On the stabilization of the wave equa tion," in Analysis and Optimization of Systems : State and Frequency Domain Approaches for Infinite Dimensional Systems, R. F. Curtain, ed., Lecture Notes in Control and Information Sciences, Vol. 185, SpringerVerlag, 1993, pp. 531-543.

[14] Morgül, Ö., "Dynamic boundary control of a rotating flexible structure," Proceedings of the 31st IEEE Conference on Decision and Control, Tucson, Arizona, Dec. 1992, pp. 1313-1314.

[15] Pazy, A., Semigroups of Linear Operators and Applications to Partial Differential Equations, New York, Springer-Verlag, 1983.

[16] Slotine, J. J. E , W. Pi, Applied Nonlinear Control, Englewood Cliffs, New Jersey: Prentice-Hall, 1991. 